\title{
Unfallmeldungen zu Nadelstichverletzungen bei Beschäftigten in Krankenhäusern, Arztpraxen und Pflegeeinrichtungen
}

\author{
Workers' Compensation Claims for Needlestick Injuries Among
} Healthcare Personnel in Hospitals, Doctors' Surgeries and Nursing Institutions

() (1) $\circledast \Theta$

Madeleine Dulon, Birgit Lisiak, Dana Wendeler, Albert Nienhaus

Institut

Arbeitsmedizin, Gefahrstoffe und Gesundheitswissenschaften, Berufsgenossenschaft für Gesundheitsdienst und Wohlfahrtspflege (BGW), Hamburg

Schlüsselwörter

Nadelstichverletzung, Gesundheitsberufe, TRBA 250,

Sicherheitsgeräte

Key words

needlestick injuries, health personnel, technical rule 250, safety engineered devices

\section{Bibliografie}

DOI https://doi.org/10.1055/s-0043-114003

Online-Publikation: 28.7.2017

Gesundheitswesen 2018; 80: 176-182

(c) Georg Thieme Verlag KG Stuttgart · New York ISSN 0941-3790

\section{Korrespondenzaddresse}

Dr. Madeleine Dulon

Arbeitsmedizin, Gefahrstoffe und Gesundheitswissenschaften Berufsgenossenschaft für Gesundheitsdienst und Wohlfahrtspflege (BGW)

Pappelallee 33/35/37

22089 Hamburg

Madeleine.Dulon@bgw-online.de

\section{ZUSAMMENFASSUNG}

Ziel Mit der aktualisierten „Technischen Regel für biologische Arbeitsstoffe im Gesundheitsdienst und in der Wohlfahrtspflege“ (TRBA 250) sind Schutzmaßnahmen zur Prävention von Stich- und Schnittverletzungen seit März 2014 in Deutschland neu geregelt. In der vorliegenden Studie wird 6 Monate nach Inkrafttreten der TRBA 250 untersucht, ob sich Krankenhäuser, Arztpraxen und Pflegeeinrichtungen unterscheiden hinsichtlich der Ursachen von Nadelstichverletzungen (NSV) sowie der Verfügbarkeit und Anwendung von stichsicheren Instrumenten (SSI).
Methode Im Rahmen dieser Studie wurde eine Stichprobe von Unfallmeldungen ausgewertet, die im Zusammenhang mit einer NSV bei der Berufsgenossenschaft für Gesundheitsdienst und Wohlfahrtspflege (BGW) angezeigt wurden ( $n=1602)$. Zum Unfallhergang und zum Umgang mit SSI an den Arbeitsplätzen wurden die Beschäftigten telefonisch befragt (Responsequote 33,3\%). Die Auswertung erfolgte deskriptiv, getrennt für 3 Tätigkeitsbereiche: Krankenhäuser, Arztpraxen und Pflegeeinrichtungen, worunter stationäre Altenpflege und ambulante Dienste zusammengefasst wurden.

Ergebnisse In allen 3 Bereichen ereignete sich etwa die Hälfte der NSV nicht bei der invasiven Prozedur, sondern erst im Anschluss daran bei der Entsorgung der Geräte. Kanülen für subkutane Injektionen waren die Ursache für $30 \%$ aller NSV, in Pflegeeinrichtungen lag der Anteil bei über $50 \%$. SSI waren in Krankenhäusern und Arztpraxen an $80 \%$ und in Pflegeeinrichtungen an $50 \%$ der Arbeitsplätze vorhanden. Als Grund für die NSV im Zusammenhang mit einem SSI wurde in über $90 \%$ der Fälle fehlende Erfahrung in der Aktivierung des Sicherheitsmechanismus angegeben. SSI waren an $20 \%$ der NSV in Krankenhäusern und Arztpraxen beteiligt und an $10 \%$ der NSV in Pflegeeinrichtungen.

Schlussfolgerung Schulungen zur sicheren Entsorgung von spitzen Instrumenten sollten sich an alle Berufsgruppen wenden, die in Kontakt mit solchen Instrumenten kommen könnten. Das Infektionsrisiko durch subkutane Kanülen ist derzeit nicht eindeutig bestimmbar. Gleichwohl sind in der stationären Altenpflege und in der ambulanten Versorgung Schulungen zum sicheren Umgang mit Pen-Kanülen notwendig in Anbetracht des hohen Anteils an Unfällen, die in diesen Tätigkeitsbereichen durch subkutane Injektionsnadeln verursacht wurden.

\section{ABSTRACT}

Objective The "Technical rules for biological agents in healthcare and welfare facilities" (TRBA 250) came into force in March 2014 in Germany and deals with protective measures to prevent needlestick injuries (NSI). The present study covers the period of 6 months after TRBA 250 came into force. The study had 2 objectives: to investigate whether hospitals, doctors' 
surgeries and care facilities differ with respect to the causes of needlestick injuries (NSI) and to collect data on availability and implementation of safety-engineered devices (SED) in these healthcare settings workplaces.

Methods This study analyses workers' compensation claims for NSI, as received by the Institution for Statutory Accident Insurance and Prevention in the Health and Welfare Services (Berufsgenossenschaft für Gesundheitsdienst und Wohlfahrtspflege, BGW) $(n=1602)$. During a telephone interview (response rate $33.3 \%$ ), the subjects were asked about the course of the accident and the handling of SED at the workplace. Descriptive analyses were performed for hospitals, doctors' surgeries and care facilities (including inpatient care for the elderly and outpatient care).

Results In all 3 settings, about half of the NSI did not occur during the invasive procedure, but during the subsequent disposal of the instruments. $30 \%$ of all NSI were caused by needles for subcutaneous injections; in care facilities, the proportion was above $50 \%$. SED were involved in $20 \%$ of the NSI in hospitals and doctors' surgeries and in $10 \%$ of NSI in care facilities. Lack of experience in activating the safety mechanism was the most important cause of failure for NSI with SED. SED were available at the workplace in $80 \%$ of hospitals and doctors' surgeries and in $50 \%$ of care facilities.

Conclusion Training on the safe disposal of sharp instruments should be provided to all professional groups who come into contact with such instruments. It is currently not possible to provide a reliable estimate of the risk of infection from subcutaneous needles. As a high proportion of NSI in nursing homes and outpatient care services were caused by subcutaneous needles, training on safe handling practices for disposal of needles is needed in these settings.

\section{Einleitung}

Die EU-Richtlinie 2010/32 zur Vermeidung von NSV legte Mindestanforderungen zur Prävention fest und verlangte von den EU-Mitgliedern die Umsetzung in nationales Recht [1]. In Deutschland geschah dies im Rahmen der Novellierung der Biostoffverordnung in 2013. Die Technischen Regeln für Biologische Arbeitsstoffe (TRBA) geben den Stand der Technik, Arbeitsmedizin und Arbeitshygiene sowie sonstige gesicherte wissenschaftliche Erkenntnisse für Tätigkeiten mit biologischen Arbeitsstoffen wieder. Die TRBA 250 „Biologische Arbeitsstoffe im Gesundheitswesen und in der Wohlfahrtspflege“" konkretisiert im Rahmen ihres Anwendungsbereichs die Anforderungen der Biostoffverordnung. Bei Einhaltung der Technischen Regeln kann der Arbeitgeber davon ausgehen, dass die entsprechenden Anforderungen der Verordnung erfüllt sind. Mit der Überarbeitung der TRBA 250 sind die Maßnahmen zum Schutz von Beschäftigten vor Infektionsgefahren seit März 2014 im Gesundheitsdienst anzuwenden. Um Beschäftigte im Gesundheitsdienst vor Verletzungen mit spitzen medizinischen Instrumenten zu schützen, ist die Verwendung von stichsicheren Instrumenten (SSI) nach der TRBA 250 immer dann vorgeschrieben, wenn mit der Übertragung infektionsrelevanter Blutmengen gerechnet werden muss [2]. Weitere Schutzmaßnahmen der TRBA 250 sind z. B. das Verbot, gebrauchte Kanülen in die Schutzkappe zurückzustecken (sog. Recapping), und die Entsorgung spitzer medizinischer Instrumente in ausschließlich dafür vorgesehenen Behältern [2].

Nadelstichverletzungen (NSV) zählen zu den häufigsten Arbeitsunfällen von Beschäftigten im Gesundheitsdienst. Verletzungen an mit Blut kontaminierten Kanülen bedeuten für die Betroffenen eine ernst zu nehmende Gefährdung durch blutübertragene Infektionen [3-5]. Die Angaben zur Häufigkeit von NSV variieren zwischen 1,4 und 9,5 pro 100 pflegerisch und/oder medizinisch tätigen Beschäftigten pro Jahr [6]. Schätzungen für Deutschland gehen davon aus, dass vor Einführung der SSI jährlich ca. 500000 NSV auftraten [7]. Daten der Berufsgenossenschaft für Gesundheitsdienst und Wohlfahrtspflege (BGW) zeigen, dass die Anzahl der jährlich angezeigten Arbeitsunfälle im Zusammenhang mit einer NSV von 37000 im Jahr 2007 auf 51000 im Jahr 2015 angestiegen ist ( Abb. 1) [8]. Die Aufwendungen lagen in den ersten 12 Monaten nach der NSV bei $110 €$ pro Fall für serologische Tests, medizinische Behandlung und Postexpositionsprophylaxe. Neben diesen direkten Kosten fallen durch NSV noch indirekte Kosten bei den betroffenen Unternehmen sowie monetär nicht quantifizierbare Auswirkungen wie psychische Belastungen bei den Beschäftigten an [9].

In dieser Studie wurde untersucht, ob sich Krankenhäuser, Arztpraxen und Pflegeeinrichtungen hinsichtlich der Ursachen von Häufigkeit sowie der Verfügbarkeit und Anwendung von stichsicheren Instrumenten (SSI) unterscheiden.

\section{Methodik}

Die Studienpopulation bestand aus Beschäftigten, von denen eine NSV als Arbeitsunfall gemeldet worden war. Die Unfallmeldungen wurden bei 4 vorab ausgewählten Bezirksverwaltungen der BGW gesammelt. Als Einschlusskriterium galt ein Eingangsdatum zwischen dem 1. und 31. November 2014. Die Unfallmeldungen wurden dem Studienteam zur Verfügung gestellt. Der Unfallmeldung konnten Angaben zum Unfall sowie zu Name, Adresse und Telefonnummer der verletzten Person entnommen werden. Datenschutzrechtliche Aspekte beim Umgang mit den personenbezogenen Angaben wurden mit dem Datenschutzbeauftragten der BGW abgestimmt.

Eine NSV wurde in dieser Studie definiert als jegliche Stich- oder Schnittverletzung der Haut durch medizinische Geräte, die mit potenziell infektiösem Material kontaminiert waren. Unfallmeldungen, die diese Einschlusskriterien erfüllten, bildeten die Grundlage für die vorliegende Auswertung. Um differenzierte Angaben zum Unfallhergang und zur Ausstattung der Arbeitsplätze mit SSI zu erhalten, wurden die Beschäftigten unter ihrer privaten Telefonnummer angerufen und um ein Telefoninterview gebeten. Vor Beginn des Interviews wurde darüber informiert, dass die Teilnahme an der Studie freiwillig ist und alle Angaben anonym und ohne Bezug zu der Einrichtung ausgewertet werden. Für die Auswertung wurden die Arbeitsplätze 3 Tätigkeitsbereichen zugeordnet: 1. Krankenhäuser (alle Fachrichtungen, Universitätskliniken, Reha-Kliniken und Dialysen); 2. Arztpraxen (alle Fachrichtungen; Zahnarztpraxen und Laboratorien); 3. Pflegeeinrichtungen (Einrichtungen der stationären Altenpflege und anderer Langzeitpflege sowie der ambulan- 


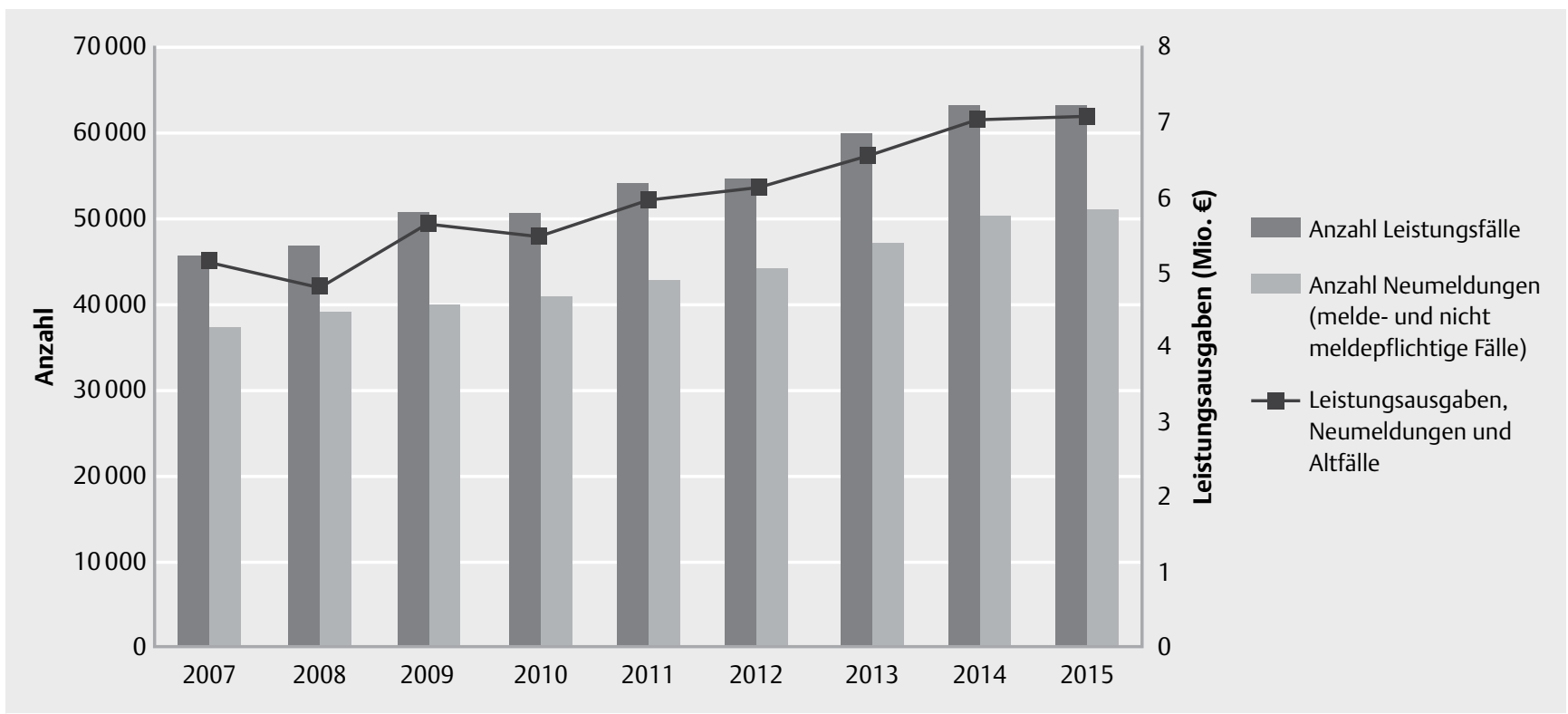

Abb. 1 Nadelstichverletzungen ${ }^{1}$ und Leistungsausgaben ${ }^{2}$ (Daten der BGW).

1 Arbeitsunfälle durch einen spitzen Gegenstand, verbunden mit einer oberflächlichen Verletzung an der Hand, bei denen postexpositionelle Maßnahmen verordnet wurden.

2 Labor- und Impfkosten; Aufwendungen für die antivirale Therapie bei Transmission sind nicht enthalten.

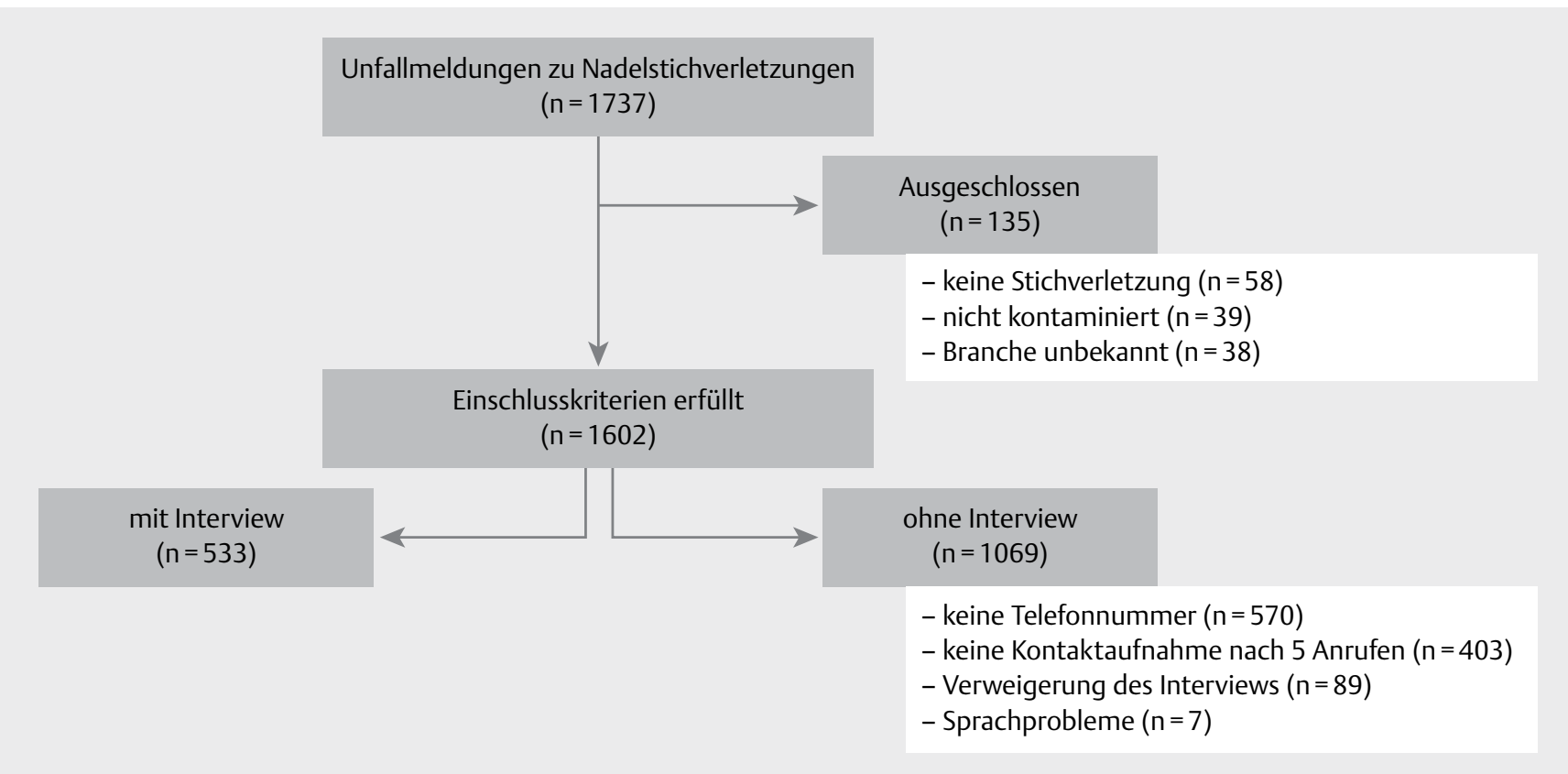

Abb. 2 Fluss-Diagramm zu ein- und ausgeschlossenen Fällen - Unfallmeldungen zu Nadelstichverletzungen bei der BGW.

ten Versorgung). Die unfall- und arbeitsplatzbezogenen Merkmale wurden mittels $95 \%$-Konfidenzintervall (95\%-KI) für Proportionen beschrieben. Bei Nicht-Überschneidung der KI wurden Unterschiede als statistisch signifikant gewertet. Die Berechnung des 95\%-KI erfolgte nach der Wilson-Score-Methode unter Verwendung der OpenSource Software OpenEpi [10]. Die Analysen wurden mit IBM SPSS Version 21.0 durchgeführt.

\section{Ergebnisse}

Während des 1-monatigen Erhebungszeitraums wurden von 1737 Beschäftigten Stichverletzungen gemeldet. 1602 Unfallmeldungen (92\%) erfüllten die Einschlusskriterien. Für ein Interview standen 533 Beschäftigte zur Verfügung. Mit 66,7\% der Beschäftigten war keine telefonische Kontaktaufnahme möglich ( $\triangleright$ Abb. 2). Die Teilnehmer mit Interview sind gut mit der Gesamtstichprobe vergleichbar; unter den Interviewten fanden sich lediglich für die Grup- 
\ Tab. 1 Soziodemografische Charakteristika der Beschäftigten mit Nadelstichverletzungen getrennt nach Tätigkeitsbereich, $n=1602$.

\begin{tabular}{|c|c|c|c|}
\hline & \multicolumn{3}{|c|}{ NSV pro Bereich } \\
\hline & Krankenhaus a $\mathrm{N}=954$ & Arztpraxis ${ }^{\mathrm{b}} \mathrm{N}=393$ & Pflegeeinrichtung ${ }^{\mathrm{C}} \mathrm{N}=\mathbf{2 5 5}$ \\
\hline & \multicolumn{3}{|c|}{ \% (95\%-Konfidenzintervall) } \\
\hline Geschlecht weiblich & $73,0(70,0-75,7)$ & $91,1(87,9-93,5)$ & $89,0(84,6-92,3)$ \\
\hline \multicolumn{4}{|l|}{ Alter (in Jahren) } \\
\hline$\leq 20$ & $5,8(4,5-7,4)$ & $23,7(19,7-28,1)$ & $5,9(3,6-9,5)$ \\
\hline $21-30$ & $36,8(33,8-39,9)$ & $33,1(28,6-37,9)$ & $34,9(29,3-40,9)$ \\
\hline $31-40$ & $25,0(22,3-27,8)$ & $19,6(16,0-23,8)$ & $20,0(15,6-25,3)$ \\
\hline $41-50$ & $18,6(16,2-21,1)$ & $14,0(10,9-17,8)$ & $21,2(16,6-26,6)$ \\
\hline$>50$ & $13,9(11,9-16,3)$ & $9,7(7,1-13,0)$ & $18,0(13,8-23,2)$ \\
\hline \multicolumn{4}{|l|}{ Beruf } \\
\hline Arzt & $34,1(31,1-37,1)$ & $12,0(9,1-15,5)$ & $1,2(0,4-3,4)$ \\
\hline Krankenpflegekraft & $37,9(34,9-41,1)$ & $2,0(1,0-4,0)$ & $16,9(12,8-21,9)$ \\
\hline Altenpflegekraft, Pflegehelfer & $1,9(1,2-3,0)$ & $0,0(0,0-1,0)$ & $43,9(38,0-50,0)$ \\
\hline Medizinisches Fachpersonal, MTA, ZFA & $3,4(2,4-4,7)$ & $58,3(53,3-63,0)$ & $3,5(1,9-6,6)$ \\
\hline Auszubildende & $15,6(13,5-18,1)$ & $18,3(14,8-22,5)$ & $12,9(9,4-17,6)$ \\
\hline Reinigungs-, Küchen-, Transportpersonal & $3,9(2,8-5,3)$ & $4,3(2,7-6,8)$ & $11,8(8,4-16,3)$ \\
\hline Unbekannt & $3,2(2,3-4,6)$ & $5,1(3,3-7,7)$ & $9,8(6,7-14,1)$ \\
\hline
\end{tabular}

pe der über 40-Jährigen in Pflegeeinrichtungen höhere Anteile [53 vs. $39 \%$ ]. Knapp $60 \%$ der Studienpopulation war in Krankenhäusern beschäftigt, $25 \%$ in Arztpraxen und $16 \%$ in Pflegeeinrichtungen ( $\triangleright$ Tab. 1). Beim Alter zeigte sich, dass die Nadelstichverletzten in Arztpraxen signifikant jünger waren als in den beiden anderen Bereichen. Aus Krankenhäusern und Pflegeeinrichtungen wurden die meisten NSV von Pflegekräften gemeldet; aus Arztpraxen stammten die meisten Meldungen von medizinischem Fachpersonal. Personal aus pflegefernen Berufsgruppen hatte sich in Pflegeeinrichtungen signifikant häufiger an medizinischen Instrumenten verletzt als in den beiden anderen Bereichen.

\section{Unfallursachen}

In Pflegeeinrichtungen wurden über $50 \%$ der NSV durch subkutane Kanülen verursacht ( $\triangleright$ Tab. 2). In Krankenhäusern waren diese ebenfalls häufig an NSV beteiligt, gefolgt von intravenösen Kanülen und chirurgischen Instrumenten. In Arztpraxen wurden die meisten NSV durch intravenöse Kanülen verursacht, allerdings fehlte für ein Fünftel der Kanülen eine Angabe zur Injektionsart. Die Tätigkeit, bei der es zur NSV kam, war für etwa ein Drittel der Fälle bekannt. Die meisten NSV ereigneten sich im Zusammenhang mit der Entsorgung. In Arztpraxen und Pflegeeinrichtungen kam es bei der Entsorgung signifikant häufiger zu NSV als in Krankenhäusern. Kapillare Blutentnahmen und Verletzungen beim Aufräumen durch unsachgemäß abgelegte Kanülen waren weitere Tätigkeiten, bei denen es in Pflegeeinrichtungen gehäuft zu NSV kam. Bei Beschäftigten in Krankenhäusern ereignete sich ein Großteil der NSV im Zusammenhang mit chirurgischen Prozeduren oder beim Hantieren mit Instrumenten.

Von über der Hälfte der telefonisch Befragten wurde persönliche Faktoren wie fehlende Aufmerksamkeit und Ablenkung als Ursache für die NSV angeführt ( $\triangleright$ Tab. 3). Für gut ein Drittel der
Unfälle wurden Umstände angeführt, die nicht mit dem invasiven Vorgang im Zusammenhang standen, sondern sich im Anschluss daran ereigneten und auf organisatorische Mängel zurückgeführt wurden.

\section{Nadelstichverletzungen und Maßnahmen zu deren Vermeidung}

Einige Mindestanforderungen zur Vermeidung von NSV wurden in Arztpraxen und signifikant seltener erfüllt als in den Krankenhäusern. Dies betraf v. a. die Ausstattung der Arbeitsplätze mit SSI in Pflegeeinrichtungen ( $\triangleright$ Tab. 3). Auch die standardmäßige Verwendung von SSI für die Blutentnahme und zur Behandlung von als infektiös bekannten Patienten war in Pflegeeinrichtungen seltener vorgegeben ( $>$ Tab. 3). In Arztpraxen waren SSI beim Legen von Gefäßkathetern seltener vorgesehen als in Krankenhäusern. Eine schriftliche Betriebsanweisung zur Verwendung von SSI gab es für zwei Drittel der Arbeitsplätze. Instrumente mit einem Sicherheitsmechanismus waren in Krankenhäusern und Arztpraxen häufiger an NSV beteiligt als in Pflegeeinrichtungen. Von den Beschäftigten, an deren Arbeitsplätzen SSI vorhanden waren, hatten weniger als ein Drittel ein SSI verwendet, als es zu der NSV kam. Die Mehrheit der verwendeten Kanülen war mit einem Sicherheitsmechanismus ausgestattet, der vom Anwender aktiv ausgelöst werden musste. Die überwiegende Mehrheit der Anwender führte den Unfall auf eine unzureichende Erfahrung bei der Handhabung der SSI zurück.

An etwa der Hälfte der Arbeitsplätze, die mit SSI ausgestattet waren, gab es Schulungen zu deren Handhabung. Teilnehmer, die sich an einem SSI verletzt hatten, hatten in 70 (64\%) von 110 Fällen die Möglichkeit, an ihrem Arbeitsplatz an einer SSI-Schulung teilzunehmen; davon hatten 39 (56\%) in den 12 Monaten vor dem Unfall eine Schulung besucht. 
- Tab. 2 Unfallverursachendes Instrument und Art der Tätigkeit von Beschäftigten mit Nadelstichverletzung (NSV) getrennt nach Tätigkeitsbereicha , $\mathrm{n}=1602$.

\begin{tabular}{|c|c|c|c|}
\hline & \multicolumn{3}{|c|}{ NSV pro Bereich } \\
\hline & Krankenhaus N= 954 & Arztpraxis $\mathrm{N}=393$ & Pflegeeinrichtung $\mathrm{N}=255$ \\
\hline Instrument bekannt & $766(80,3 \%)$ & 291 (74,1\%) & $227(89,0 \%)$ \\
\hline \multirow[t]{2}{*}{ Tätigkeit bekannt } & $372(39,0 \%)$ & $140(35,6 \%)$ & $95(24,2 \%)$ \\
\hline & \multicolumn{3}{|c|}{ \% (95\%-Konfidenzintervall) } \\
\hline Instrument & $(n=766)$ & $(n=291)$ & $(n=227)$ \\
\hline $\begin{array}{l}\text { i.v. Kanüle (Blutentnahme-, Venenkatheterkanüle, } \\
\text { Butterfly) }\end{array}$ & $23,5(20,6-26,6)$ & $24,7(20,1-30,0)$ & $8,4(5,4-12,7)$ \\
\hline i.m. Kanüle & $1,6(0,9-2,7)$ & $5,8(3,7-9,2)$ & $1,3(0,5-3,8)$ \\
\hline s.c. Kanüle (einschließlich Pen-Injektionskanüle) & $30,2(27,0-33,5)$ & $13,1(9,7-17,4)$ & $53,8(47,3-60,1)$ \\
\hline Kanüle (Injektionsart unbekannt) & $10,3(8,4-12,7)$ & $20,3(16,1-25,3)$ & $11,0(7,6-15,8)$ \\
\hline Lanzette & $2,0(1,2-3,2)$ & $2,8(1,4-5,3)$ & $25,6(20,3-31,6)$ \\
\hline Skalpell & $9,3(7,4-11,5)$ & $11,4(8,2-15,5)$ & $0,0(0,0-1,7)$ \\
\hline Sonstige chirurgische Instrumente (inkl. Nähnadel) & $23,2(20,4-26,4)$ & $22,0(17,6-27,1)$ & $0,0(0,0-1,7)$ \\
\hline Tätigkeit & $(n=372)$ & $(n=140)$ & $(n=95)$ \\
\hline Blutentnahme & $7,5(5,3-10,7)$ & $6,4(3,4-11,8)$ & $0,0(0,0-3,9)$ \\
\hline Injektion i.v., i.m., s.c. & $11,0(8,2-14,6)$ & $12,1(7,7-18,6)$ & $17,9(11,5-26,8)$ \\
\hline Legen/entfernen von Gefäßzugängen & $12,6(9,6-16,4)$ & $5,7(2,9-10,9)$ & $4,2(1,7-10,3)$ \\
\hline Kapillarblutentnahme Finger & $0,3(0,05-1,5)$ & $1,4(0,4-5,1)$ & $14,7(9,0-23,2)$ \\
\hline Aufräumen ${ }^{\mathrm{b}}$ & $8,1(5,7-11,3)$ & $9,3(5,5-15,2)$ & $13,7(8,2-22,0))$ \\
\hline Entsorgung $^{c}$ & $19,9(16,2-24,3)$ & $38,6(30,9-46,8)$ & $38,9(29,8-49,0)$ \\
\hline Chirurgische Prozedur (inkl. Nähen) & $28,0(23,6-32,7)$ & $14,3(9,4-21,0)$ & $0,0(0,0-3,9)$ \\
\hline Hantieren mit Instrumenten & $12,6(9,6-16,4)$ & $12,1(7,7-18,6)$ & $10,5(5,8-18,3)$ \\
\hline
\end{tabular}

\section{Diskussion}

Zu den Ursachen von NSV wurden in den vergangenen 15 Jahren zahlreiche Studien durchgeführt. Die meisten davon bezogen sich auf die Situation an Universitätskliniken und standen im Zusammenhang mit der Einführung von SSI [11-14]. Mit der Situation in nicht-klinischen Tätigkeitsbereichen haben sich dagegen nur wenige Untersuchungen beschäftigt $[15,16]$. Die vorliegende Studie zeigt, dass in nicht-klinischen Bereichen solche Instrumente für NSV ursächlich sind, die aufgrund des Krankheits- und Behandlungsspektrums in dem jeweiligen Bereich häufig verwendet werden subkutane Kanülen und Lanzetten in Einrichtungen der stationären Altenpflege und der ambulanten Dienste sowie Kanülen von Blutentnahmesystemen in Arztpraxen. NSV bei der Entsorgung von Instrumenten ereigneten sich in nicht-klinischen Bereichen sehr viel häufiger als in Krankenhäusern. Diese Zahl erhöht sich noch um etwa 8-13 Prozentpunkte (je nach Bereich), wenn statt der Angaben aus der Unfallmeldung die telefonisch zum Unfallhergang erhobenen Angaben herangezogen werden [17]. Dass für Reinigungsund Transportkräfte in nicht-klinischen Bereichen ein erhöhtes Risiko besteht, sich an unsachgemäß abgelegten Kanülen zu verletzten, wird auch von anderen Autoren berichtet [18].

\section{Ausstattung mit stichsicheren Instrumenten}

Für die Vermeidung von Arbeitsunfällen waren an den betroffenen Arbeitsplätzen in Krankenhäusern und Arztpraxen gute Voraussetzungen gegeben: Die Quote der Verfügbarkeit von SSI lag zwischen 70 und $80 \%$ und ihre Verwendung war - nach den Angaben der Be- fragten - für die in der TRBA 250 erwähnten infektionsrelevanten Prozeduren auch vorgesehen. Anhand des Unfallhergangs lässt sich allerdings zeigen, dass nur weniger als ein Drittel der Teilnehmer die SSI auch verwendet hat. Die von den SSI-Anwendern verwendeten Formulierungen zur Beschreibung der Unfallursache deuten darauf hin, dass es bei der Auslösung des Sicherheitsmechanismus Probleme gab. Diese Vermutung passt zu den Beobachtungen anderer Autoren, die die Inhalte von Abwurfbehältern untersucht haben, wonach der Sicherheitsmechanismus von vielen Kanülen nur unvollständig aktiviert war $[19,20]$. Passiv wirkende Sicherheitsmechanismen scheinen einen besseren Schutz vor NSV zu bieten als aktiv auszulösende [11, 21, 22]. Allerdings geben andere Autoren zu bedenken, dass es für diesen Zusammenhang insgesamt nur eine geringe Evidenz gibt [23].

Schulungen zur Handhabung von SSI wurden nur an zwei Dritteln der Arbeitsplätze angeboten. Zum Inhalt der Schulungen können wir keine Angaben machen. Nach Aussagen derjenigen, die sich bei Verwendung SSI verletzt haben, hatte nur ein Drittel in den 12 Monaten vor dem Unfall an einer Schulung teilgenommen. Die von den SSI-Anwendern verwendeten Formulierungen deuten jedoch darauf hin, dass bei Stress, Überarbeitung oder unerwarteten Ereignissen der Umgang mit dem Sicherheitsmechanismus nicht gelingt.

Nach Einführung von SSI wird in der Literatur übereinstimmend von einem Rückgang bei NSV berichtet [13, 14, 22, 24]. Allerdings kann es sich hierbei auch um einen Publikationsbias handeln oder um eine Überschätzung des Effekts, da die Beobachtungszeit nach Einführung der SSI jeweils nur einige Monate umfasste. Auf Basis 
- Tab. 3 Unfallursache und arbeitsplatzbezogene Merkmale von Beschäftigten mit Nadelstichverletzungen (NSV) durch stichsichere Instrumente (SSI) getrennt nach Tätigkeitsbereicha, Interviewgruppe $(n=533)$.

\begin{tabular}{|c|c|c|c|}
\hline & \multicolumn{3}{|c|}{ NSV pro Bereich } \\
\hline & Krankenhaus $\mathrm{N}=\mathbf{3 0 0}$ & Arztpraxis $\mathrm{N}=139$ & Pflegeeinrichtung $\mathrm{N}=94$ \\
\hline & \multicolumn{3}{|c|}{ \% (95\% Konfidenzintervall) } \\
\hline \multicolumn{4}{|l|}{ Unfallursache $^{\mathrm{b}}$} \\
\hline Aufmerksamkeit unzureichend & $60,0(54,4-65,4)$ & $65,5(57,2-72,9)$ & $48,9(39,1-58,9)$ \\
\hline Organisatorische Mängel $^{\mathrm{c}}$ & $36,3(31,1-41,9)$ & $33,1(25,8-41,3)$ & $40,4(31,1-50,5)$ \\
\hline Unerwartete Bewegung des Patienten & $12,7(9,4-16,9)$ & $7,2(4,0-12,7)$ & $10,6(5,9-18,5)$ \\
\hline Ablenkung durch Umgebung & $7,3(4,9-10,9)$ & $2,9(1,1-7,2)$ & $5,3(2,3-11,9)$ \\
\hline Technisches Problem/Produktversagen & $6,0(3,8-9,3)$ & $5,8(2,9-11,0)$ & $8,5(4,4-15,9)$ \\
\hline SSI am Arbeitsplatz vorhanden & $82,7(78,0-86,5)$ & $75,5(67,8-81,9)$ & $50,0(40,1-59,9)$ \\
\hline SSI beim Unfall verwendet & $23,6(19,2-28,8)$ & $21,6(15,9-29,1)$ & $9,6(5,1-17,2)$ \\
\hline Arbeitsplätze mit SSI & $(n=248)$ & $(n=105)$ & $(n=47)$ \\
\hline \multicolumn{4}{|l|}{ Verwendung von SSI als Standard bei } \\
\hline Blutentnahme & $78,6(73,1-83,3)$ & $74,3(65,2-81,7)$ & $40,4(27,6-54,7)$ \\
\hline Behandlung von bekannt infektiösen Patienten & $72,6(66,7-77,8)$ & $68,6(59,2-76,7)$ & $46,8(33,3-60,8)$ \\
\hline Legen von Gefäßkathetern & $71,0(65,0-76,3)$ & $47,6(38,3-57,1)$ & $0,0(0,0-7,6)$ \\
\hline Betriebsanweisung zur Verwendung von SSI vorhanden & $59,7(53,5-65,6)$ & $74,3(65,2-81,7)$ & $59,6(45,3-72,4)$ \\
\hline Schulung zu SSI wird angeboten & $54,8(48,6-60,9)$ & $58,1(48,5-67,1)$ & $59,6(45,3-72,4)$ \\
\hline Unfälle bei Verwendung SSI & $(n=71)$ & $(n=30)$ & $(n=9)$ \\
\hline Sicherheitsmechanismus: selbstauslösend & $18,3(11,0-28,9)$ & $16,7(7,4-33,6)$ & $44,5(18,9-73.4)$ \\
\hline \multicolumn{4}{|l|}{ Ursacheb für den Unfall durch SSI } \\
\hline Praktische Erfahrung unzureichend & $93,0(84,6-97,0)$ & $100(88,7-100)$ & $100,0(70,1-100,0)$ \\
\hline Sicherheitsmechanismus defekt & $23,9(15,5-35,0)$ & $16,7(7,4-33,6)$ & $11,1(2,0-43,5)$ \\
\hline Schulung zu SSI wird angeboten & $62,0(50,3-72,4)$ & $66,7(48,8-80,8)$ & $66,7(35,4-87,9)$ \\
\hline
\end{tabular}

der vorliegenden Untersuchung kann festgestellt werden, dass 6 Monate nach Inkrafttreten der TRBA 250 die Umsetzung der Schutzmaßnahmen zur Prävention von NSV in stationären Altenpflegeeinrichtungen, ambulanten Diensten und Arztpraxen noch unvollständig erfolgte.

\section{Subkutane Kanülen}

Kanülen für subkutane Injektionen wurden auch von anderen Autoren als eine der Hauptursachen für NSV identifiziert [15, 19, 21]. Bislang scheinen Kanülen mit Sicherheitsmechanismus eher selten bei subkutanen Injektionen eingesetzt zu werden. Das kann daran liegen, dass der Einsatz von SSI dafür von der EU-Richtlinie nicht explizit empfohlen wird. Die WHO spricht sich hingegen für die Verwendung von Instrumenten mit Sicherheitsmechanismus für subkutane Injektionen aus und begründet dies mit der Vielzahl an subkutanen Applikationen, die weltweit vorgenommen werden [25]. Zur Wirksamkeit der SSI in Bezug auf die Reduzierung von NSV bei subkutanen Injektionen sind die Studienergebnisse widersprüchlich [23, 26]. Die Autoren der Übersichtsarbeit, die der WHO-Richtlinie zugrunde liegt, gehen von einer moderaten Evidenz aus, dass durch die Verwendung von SSI bei subkutanen Injektionen NSV verhindert werden können [26]. In Deutschland wird eine Empfehlung für die Verwendung von SSI für subkutane Injektionen noch diskutiert. Ob diese zukünftig auch in der TRBA 250 explizit empfohlen wird, ist ungewiss. Die Abschätzung der Infektionsgefährdung durch subkutane Injektionen ist schwierig. Aus Studien liegen keine validen Daten hinsichtlich des Risikos der Übertragung einer Infektion nach einer NSV mit einer subkutanen Kanüle vor [26]. Dass eine Serokonversion nach Verletzung an subkutanen Kanülen allerdings möglich ist, zeigen gut dokumentierte Kasuistiken aus Frankreich (4 Fälle einer Hepatitis C-Infektion im Zeitraum von 1991 bis 2005) [27].

\section{Limitation}

Im Rahmen der Telefoninterviews konnten wir nicht klären, ob die Befragten mit dem Begriff „Instrumente mit Sicherheitsmechanismus" vertraut waren. Fehlklassifizierungen sind deshalb möglich.

\section{FAZIT}

- In Arztpraxen, Einrichtungen der stationären Altenpflege und des ambulanten Dienstes sollte in allen Berufsgruppen das Bewusstsein für die Gefahren bei der Entsorgung von Kanülen geschaffen werden.

- Es sind weitere Anstrengungen zur Vermeidung von NSV durch subkutane Kanülen erforderlich. 


\section{Interessenskonflikt}

Die Autoren geben an, dass keine Interessenskonflikte bestehen.

\section{Literatur}

[1] Europäisches Parlament. Richtlinie 2010/32/EU DES RATES vom 10. Mai 2010 zur Durchführung der von HOSPEEM und EGÖD geschlossenen Rahmenvereinbarung zur Vermeidung von Verletzungen durch scharfe/spitze Instrumente im Krankenhaus- und Gesundheitssektor. Amtsblatt der Europäischen Union 2010; L134: 66-72

[2] Ausschuss für Biologische Arbeitsstoffe (ABAS). Technische Regeln für Biologische Arbeitsstoffe. Biologische Arbeitsstoffe im Gesundheitswesen und in der Wohlfahrtspflege (TRBA 250). GMBI 2014, Nr. 10/11 vom 27.3.2014.

[3] Prüss-Üstun A, Rapiti E, Hutin Y. Estimation of the global burden of disease attributable to contaminated sharps injuries among health-care workers. Am J Ind Med 2005; 48: 482-490

[4] Siegmann S, Muth T, Kluth $W$ et al. Nadelstichverletzungen bei Studierenden der Humanmedizin. Gesundheitswesen 2016; 78: 22-27

[5] Wicker S, Rabenau HF, Schachtrupp A et al. Arbeitsbedingte virale Infektionen in der Pflege- eine Umfrage. Gesundheitswesen 2016, doi:10.1055/s-0042-116317

[6] Elseviers MM, Arias-Guillen M, Gorke A et al. Sharps injuries amongst healthcare workers: review of incidence, transmissions and costs. J Ren Care 2014; 40: 150-156

[7] Hofmann F, Kralj N, Beie M. Kanülenstichverletzungen im Gesundheitsdienst - Häufgkeit, Ursachen und Präventionsstrategien. Gesundheitswesen 2002; 64: 259-266

[8] Berufsgenossenschaft für Gesundheitsdienst und Wohlfahrtspflege (BGW). Anzahl der jährlich angezeigten Arbeitsunfälle im Zusammenhang mit einer Nadelstichverletzung mit Leistungsausgaben. Persönliche Mitteilung der Abteilung Reha Koordination/Statistik und Controlling. 2017

[9] Mannocci A, De Carli G, Di Bari V et al. How much do needlestick injuries cost? A systematic review of the economic evaluations of needlestick and sharps injuries among healthcare personnel. Infect Control Hosp Epidemiol 2016; 37: 635-646

[10] Dean AG, Sullivan KM, Soe MM. OpenEpi: Open source epidemiologic statistics for public health, Version 3.1. 2013; Im Internet: www. OpenEpi.com Stand: 06.04.2013. Zugriff: 23.02.2017

[11] Hoffmann C, Buchholz L, Schnitzler P. Reduction of needlestick injuries in healthcare personnel at a university hospital using safety devices. J Occup Med Toxicol 2013; 8: 20

[12] Müller-Barthelmeh R, Buchholz L, Nübling M et al. Quality control of devices with needle protection technology. Intervention study on the reduction of needlestick injuries by using instruments with needleprotection technology. Arbeitsmed Sozialmed Umweltmed 2006; 41: 210-217
[13] Wicker S, Gottschalk R, Hofmann F et al. Effectiveness and efficiency of safety devices. Arbeitsmed Sozialmed Umweltmed 2010; 45: 428-433

[14] Fukuda H, Yamanaka N.. Reducing needlestick injuries through safety-engineered devices: results of a Japanese multi-centre study. J Hosp Infect 2016; 92: 147-153

[15] Kiss P, De Meester M, Braeckman L. Needlestick injuries in nursing homes: the prominent role of insulin pens. Infect Control Hosp Epidemiol 2008; 29: 1192-1194

[16] Shah SM, Bonauto D, Silverstein B et al. Workers' compensation claims for needlestick injuries among healthcare workers in Washington State, 1996-2000. Infect Control Hosp Epidemiol 2005; 26: 775-781

[17] Dulon M, Lisiak B, Wendeler D et al. Causes of needlestick injuries in three healthcare settings: analysis of accident notifications registered six months after the implementation of EU Directive 2010/32/EU in Germany. J Hosp Infect 2017; 95: 306-311

[18] Blenkharn Jl, Odd C.. Sharps injuries in healthcare waste handlers. Ann Occup Hyg 2008; 52: 281-286

[19] Lee JM, Botteman MF, Nicklasson L et al. Needlestick injury in acute care nurses caring for patients with diabetes mellitus: a retrospective study. Curr Med Res Opin 2005; 21: 741-747

[20] Mendelson MH, Lin-Chen BY, Solomon R et al. Evaluation of a safety resheathable winged steel needle for prevention of percutaneous injuries associated with intravascular-access procedures among healthcare workers. Infect Control Hosp Epidemiol 2003; 24: 105-112

[21] Pellissier G, Migueres B, Tarantola A et al. Risk of needlestick injuries by injection pens. J Hosp Infect 2006; 63: 60-64

[22] Whitby M, McLaws ML, Slater K. Needlestick injuries in a major teaching hospital: the worthwhile effect of hospital-wide replacement of conventional hollow-bore needles. Am J Infect Control 2008; 36: 180-186

[23] Lavoie MC, Verbeek JH, Pahwa M. Devices for preventing percutaneous exposure injuries caused by needles in healthcare personnel. Cochrane Database Syst Rev 2014, doi:10.1002/14651858.CD009740.pub2 CD009740

[24] Valls V, Lozano MS, Yanez R et al. Use of safety devices and the prevention of percutaneous injuries among healthcare workers. Infect Control Hosp Epidemiol 2007; 28: 1352-1360

[25] WHO. WHO guideline on the use of safety-engineered syringes for intramuscular, intradermal and subcutaneous injections in health-care settings. 2016; Im Internet: http://apps.who.int/iris/bitstre am/10665/250144/1/9789241549820-eng.pdf. Stand: 18.04.2017

[26] Harb AC, Tarabay R, Diab B et al. Safety engineered injection devices for intramuscular, subcutaneous and intradermal injections in healthcare delivery settings: a systematic review and meta-analysis. BMC Nurs 2015; 14: 71

[27] Lot F, Abiteboul D. Contaminations professionnelles par le VIH, le VHC et le VHB chez le personnel de santé en France: Données au 31 décembre 2005. 2006; Im Internet: http://www.ladocumentationfrancaise.fr/rapports-publics/064000750-contaminations-professionnelles-par-le-vih-le-vhc-et-le-vhb-chez-le-personnel-de-sante Stand: 2006. Zugriff: 23.02.2017 